\title{
Right-handed Neutrino Dark Matter, Neutrino Masses, and non-Standard Cosmology in a $2 \mathrm{HDM}$
}

\author{
G. Arcadi, ${ }^{a, b}$ S. Profumo, ${ }^{c}$ F. S. Queiroz, ${ }^{d}$ C. \\ Siqueira ${ }^{d}$
}

${ }^{a}$ Dipartimento di Matematica e Fisica Universitá di Roma Tre, Via Della Vasca Navale 8400146 Roma, Italy

${ }^{b}$ INFN Sezione Roma Tre, Via Della Vasca Navale 8400146 Roma, Italy

${ }^{c}$ Department of Physics and Santa Cruz Institute for Particle Physics, University of California, Santa Cruz, CA 95064, USA

${ }^{d}$ International Institute of Physics, Universidade Federal do Rio Grande do Norte, Campus Universitario, Lagoa Nova, Natal-RN 59078-970, Brazil

E-mail: giorgio.arcadi@uniroma3.it,profumo@ucsc.edu, farinaldo.queiroz@iip.ufrn.br, csiqueira@iip.ufrn.br

\begin{abstract}
We explore the dark matter phenomenology of a weak-scale right-handed neutrino in the context of a Two Higgs Doublet Model. The expected signal at direct detection experiments is different from the usual spin-independent and spin-dependent classification since the scattering with quarks depends on the dark matter spin. The dark matter relic density is set by thermal freeze-out and in the presence of nonstandard cosmology, where an Abelian gauge symmetry is key for the dark matter production mechanism. We show that such symmetry allows us to simultaneously address neutrino masses and the flavor problem present in general Two Higgs Doublet Model constructions. Lastly, we outline the region of parameter space that obeys collider, perturbative unitarity and direct detection constraints.
\end{abstract}




\section{Contents}

1 Introduction $\quad 2$

2 Right-Handed Neutrino Dark Matter in a 2HDM augmented by a gauge symmetry

2.1 Yukawa Lagrangian

2.2 Gauge Anomalies

2.3 Scalar Potential

2.4 Relevant Interactions

3 Dark Matter Phenomenology

3.1 Relic Density - Standard Cosmology

3.2 Relic Density - Early Matter Domination

3.3 Direct Detection

3.4 Indirect Detection

3.5 Perturbative Unitarity

3.6 Collider bounds

3.7 Atomic Parity violation

3.8 Constraints on extra scalars

4 Results

5 Conclusions 


\section{Introduction}

There is ample evidence that the dark matter (DM) accounts for about $27 \%$ of the energy budget of our universe, i.e. $\Omega_{D M} h^{2}=0.12$, as measured with very high precision by the PLANCK Collaboration [1]. Current observations, however, do not shed light on the microscopic nature of the DM, nor to they allow to discriminate between astrophysical or particle physics solutions to the DM puzzle. Assuming a particle nature for the DM component of the Universe, it is well known that the Standard Model (SM) of particle physics cannot provide a viable candidate, which should thus be one (or more) new exotic particles. Among the many possible options, WIMPs (Weakly Interacting Massive Particles) have been regarded as one of the most promising ones, since their abundance can be elegantly accommodated, through the thermal freeze-out mechanism, by requiring $\mathrm{DM}$ masses in the $\mathrm{GeV}-\mathrm{TeV}$ range and interactions with the SM states of strength similar to weak interactions. Attempts to directly or indirectly detect WIMPs, however, have been up to now unsuccessful [2]. It should be pointed out, however, that only recently have we started to probe the "natural" parameter space of WIMPs [3].

Further evidence for new physics beyond the SM is provided by the experimental evidence of non-zero neutrino masses. As right-handed neutrinos are absent in the SM, at least in its minimal incarnation, the Higgs field cannot generate a Dirac mass term for the neutrinos. If copies of right-handed neutrinos are included in the matter content of the SM, the Yukawa coupling needed to explain neutrino masses around $0.1 \mathrm{eV}$ would be extremely, and unnaturally, suppressed. Being SM singlets, righthanded neutrinos can have as well a Majorana mass term without conflicting with gauge invariance. Given this peculiar feature of neutrino masses, New Physics beyond the SM is typically invoked for their origin. Neutrino oscillation experiments, see e.g. [4-11], have measured with great precision mass differences and mixing of the three light SM neutrinos. However, their individual masses are yet unknown and two mass orderings, normal and inverse, are allowed. Useful insight has been given, in this direction, by cosmology. Indeed measures of the Cosmic Microwave Background (CMB), constrain the sum of the neutrino masses through its effect on structure growth that comes in terms of the early Integrated Sachs Wolfe effect and gravitational lensing of the CMB [12]. In summary, they impose $\sum_{i} \nu_{i}<0.12 \mathrm{eV}$ [1]. A natural question arises at this point: can the same new physics beyond the SM be responsible, at the same time, of the generation of neutrino masses and of the DM component of the Universe? The answer is yes, and this has been driving a multitude of studies in the literature in the context of neutrino masses generated at tree-level [13-28].

As mentioned, the simplest way to generate light neutrino masses is to extend the SM with three right-handed neutrinos described by the following Lagrangian:

$$
\mathcal{L} \supset y_{a b} \overline{L_{a}} \Phi N_{b R}+\frac{M_{a}}{2} \overline{N_{a R}^{c}} N_{a R},
$$

where $\Phi$ is the SM Higgs doublet, and $M$ a Majorana bare mass term (matrix) for the right-handed neutrinos. 
After spontaneous electroweak (EW) symmetry breaking, the first term leads to a Dirac mass which mixes the left-handed and right-handed neutrinos. Three light neutrino masses are, at this point, generated through the so called (Type-I) seesaw mechanism. Since neutrino masses are given by $m_{\nu} \simeq m_{D}^{T} M^{-1} m_{D}$, with $m_{D} \ll M$ the Dirac mass term, it can be easily argued that there are multiple choices for the Yukawa couplings $y_{a b}$ and the Majorana $M$ mass that are consistent with the measured oscillation pattern and astrophysical constraints [29]. In particular, values of order one for $y_{a b}$ would imply a very high scale $M \sim 10^{12}-10^{15} \mathrm{GeV}$ for the Majorana mass term, hardly accessible to experimental tests. Furthermore, in principle one cannot accommodate a viable DM candidate in this scenario.

A common origin for DM and neutrino masses in the context of the seesaw mechanism can be achieved in a somewhat orthogonal regime, with $\mathrm{GeV}$-scale or lighter Majorana masses and comparatively small Yukawa couplings. The key aspect in this regime is the fact that the tree-level seesaw mechanism requires the presence of only two right-handed neutrinos to generate a pattern of masses and mixing of the light neutrinos compatible with laboratory tests. This leaves the freedom of assuming that the remaining right-handed neutrino be the DM candidate. In absence of additional ad-hoc symmetries, the latter would be allowed to decay into SM states. Nevertheless, it is possible to achieve a cosmologically stable state in the $\mathcal{O}(1-50) \mathrm{keV}$ mass range and very suppressed Yukawa couplings. This kind of candidate is usually referred to as sterile neutrino DM. The minimal model, which we just summarized, which accommodates both DM and neutrino masses is referred to $\nu M S M$ [30]. In this kind of scenario the DM has exceedingly suppressed interactions to be produced according the conventional freeze-out paradigm. The correct relic density can nevertheless be achieved through the so-called Dodelson-Widrow (also dubbed non-resonant) mechanism [31], consisting of production via active-sterile neutrino oscillations. A keV scale mass sterile neutrino is produced, through this mechanism, at temperatures of the order of 150 $\mathrm{MeV}$. An approximate expression for its relic density is [32-36]:

$$
\Omega_{D M} h^{2} \sim 0.1\left(\frac{\sin ^{2} \theta_{i}}{3 \times 10^{-9}}\right)\left(\frac{M_{N}}{3 \mathrm{keV}}\right)^{1.8}
$$

where $\sin ^{2} \theta_{i} \sim \sum_{a} y_{a b}^{2} v^{2} / M^{2}$, with $v$ the SM Higgs vev. It is clear that, in this setup, the right-handed neutrino is unstable due to $N \rightarrow \nu \nu \nu$, and to the loop-suppressed mode $N \rightarrow \nu \gamma$ decay. The decay width is controlled by the mixing angle and the right-handed neutrino mass, and can be observationally tested using line searches from $N$ decay, producing a line at around half the mass of the sterile neutrino, thus typically in X-rays. Searches of X-ray signals, combined with bounds from structure formation substantially exclude the parameter space corresponding to the non-resonant production mechanism for DM illustrated above (see e.g. [37, 38] for some reviews). Bounds from X-rays can be overcome by relying on the so-called Shi-Fuller [39] (resonant production) mechanism, i.e. enhanced DM production in presence of lepton asymmetry, hence requiring much smaller mixing angles to comply with the correct relic density. Tensions with structure formation are nevertheless still present [40]; no conclusive assessment can be made due to the intrinsic uncertainties of these limits. It is also 
worth pointing out that the correct relic density through resonant production implies, in the minimal model illustrated above, very tight requirements on the parameters of the new neutrino sector [41]. Alternatively, additional physics can be invoked for the production of sterile neutrinos in the early universe, see e.g. [42, 43].

In our work, we are interested in the possibility of having a weak-scale, thermally produced right-handed neutrino ${ }^{1}$. To achieve this goal, extra interactions and/or symmetries beyond those of the SM must be invoked [45-48]. More concretely, here we consider a Two Higgs Doublet Model (2HDM) augmented by a spontaneously broken, new $B-L$ Abelian gauge symmetry. This scenario aims at addressing, at the same time, the problems of flavor, dark matter and neutrino masses $[49,50]$. However, as will be shown in the following, the correct DM relic density can be hardly achieved, in the standard thermal scenario, without tension with experimental constraints. To overcome this problem, we consider the possibility of a non-standard cosmological history $^{2}$, represented by a phase of early matter domination. In summary, our work will extend previous studies in the following aspects:

(i) We consider a 2HDM augmented by a spontaneously broken additional Abelian gauge symmetry;

(ii) We consider a viable solution to the flavor problem;

(iii) We address and solve the issue of neutrino masses;

(iv) We accommodate a thermal right-handed neutrino dark matter;

(v) We explore the same setup in the context of an early matter domination period in the universe.

The paper is structured as follows: in the following Section 2, we introduce the 2HDM augmented by an additional gauge symmetry, including the relevant interactions; in Section 3, we explore the DM phenomenology, in Section 4, we discuss our results, and finally in Section 5, we present our conclusions.

\section{Right-Handed Neutrino Dark Matter in a 2HDM augmented by a gauge symmetry}

Two-Higgs doublet models (2HDM) are theoretically and experimentally appealing extensions of the SM [52-54]. One of the key features of $2 \mathrm{HDM}$ is that they do not affect the $\rho$ parameter. Moreover, $2 \mathrm{HDM}$ offer a rich environment for new physics in the sector of Higgs physics [55-60], collider searches [61], and flavor physics [6264]. Over the years, extensions to the original proposal have appeared that include

\footnotetext{
${ }^{1}$ In principle, this particle can be light, although this possibility is tightly constrained by CMB, direct and collider searches, for example, please see [44].

${ }^{2} \mathrm{~A} B-L$ extension was studied in this context in [51], exploring its testability trough gravitational waves, however, in a different approach.
} 
the introduction of additional new gauge symmetries [65-79]. Later, these new gauge symmetries were used to simultaneously solve the flavor problem in $2 \mathrm{HDM}$ and the issue of neutrino masses and mixing [50, 80-82], as well as the dark matter puzzle [14, 83-87]. It is clear that $2 \mathrm{HDM}$ augmented by gauge symmetries are gaining interest. Motivated by this, here we propose a new $2 \mathrm{HDM}$ that accommodates a thermal right-handed neutrino dark matter by adding a $B-L$ gauge symmetry. The anomaly cancellation of a new $B-L$ symmetry can be easily performed, but there are additional requirements to be considered when this symmetry is embedded in the context of a 2HDM. To understand this fact we start our discussion with the Yukawa Lagrangian. While very appealing, the $B-L$ group is not the only viable option [49]. We will thus adopt in the following a more general notation so that one can straightforwardly extend our results to the case in which the SM group is augmented by a generic $U(1)_{X}$ symmetry.

\subsection{Yukawa Lagrangian}

We will work in the context of type-I 2HDM, where only one of the scalar doublets contributes to fermion masses. This setup naturally arises via the introduction of a new gauge symmetry under which the scalar doublets $\Phi_{1}$ and $\Phi_{2}$ transform differently. In this way, the Yukawa Lagrangian reads,

$$
\begin{gathered}
-\mathcal{L}_{Y_{1}}=y_{a b}^{d} \bar{Q}_{a} \Phi_{2} d_{b R}+y_{a b}^{u} \bar{Q}_{a} \widetilde{\Phi}_{2} u_{b R}+y_{a b}^{e} \bar{L}_{a} \Phi_{2} e_{b R}+\text { h.c. }, \\
-\mathcal{L}_{Y_{2}} \supset y_{a b} \bar{L}_{a} \widetilde{\Phi}_{2} N_{b R}+y_{a b}^{M} \overline{\left(N_{a R}\right)^{c}} \Phi_{s} N_{b R}+\text { h.c. }
\end{gathered}
$$

Another Higgs field $\Phi_{s}$, singlet under the SM group but with charge $Q_{X_{s}}$ under the new gauge group (in the case of $B-L, Q_{(B-L)_{s}}=-2$ ), is introduced to spontaneously break the $U(1)_{X}$ symmetry. The corresponding vacuum expectation value (vev) will be indicated as $v_{s}$ in the following. The DM candidate is represented by the neutrino mass eigenstate $N_{1}$, which is assumed to be odd with respect to a $Z_{2}$ symmetry to ensure stability. The other two neutrinos are responsible for the generation of active neutrino masses and mixing through type-I seesaw [46, 47, 87, 88]:

$$
(\nu N)\left(\begin{array}{cc}
0 & m_{D} \\
m_{D}^{T} & M_{R}
\end{array}\right)\left(\begin{array}{c}
\nu \\
N
\end{array}\right)
$$

Taking $M_{R} \gg m_{D}$ we get $m_{\nu}=-m_{D}^{T} \frac{1}{M_{R}} m_{D}$ and $m_{N}=M_{R}$, as long as $M_{R} \gg m_{D}$, where $m_{D}=\frac{y v_{2}}{2 \sqrt{2}}$ and $M_{R}=\frac{y^{M} v_{s}}{2 \sqrt{2}}$. Using the Casas-Ibarra parametrization [89] one can straightforwardly reproduce current neutrino data (see e.g. [90]). We emphasize that the right-handed neutrino $N_{1 R}$ is decoupled by construction from this seesaw mechanism as it is odd under a $Z_{2}$ symmetry.

\subsection{Gauge Anomalies}

In order for the new $U(1)_{X}$ symmetry to be anomaly free, the following anomaly cancellation conditions must hold:

$$
\left[S U(3)_{c}\right]^{2} U(1)_{X} \rightarrow Q_{X}^{u}+Q_{X}^{d}-2 Q_{X}^{q}=0
$$




$$
\begin{gathered}
{\left[S U(2)_{L}\right]^{2} U(1)_{X} \rightarrow Q_{X}^{l}=-3 Q_{X}^{q},} \\
{\left[U(1)_{Y}\right]^{2} U(1)_{X} \rightarrow 6 Q_{X}^{e}+8 Q_{X}^{u}+2 Q_{X}^{d}-3 Q_{X}^{l}-Q_{X}^{q}=0,} \\
U(1)_{Y}\left[U(1)_{X}\right]^{2} \rightarrow-\left(Q_{X}^{e}\right)^{2}+2\left(Q_{X}^{u}\right)^{2}-\left(Q_{X}^{d}\right)^{2}+\left(Q_{X}^{l}\right)^{2}-\left(Q_{X}^{q}\right)^{2}=0, \\
{\left[U(1)_{X}\right]^{3} \rightarrow\left(Q_{X}^{e}\right)^{3}+3\left(Q_{X}^{u}\right)^{3}+3\left(Q_{X}^{d}\right)^{3}-2\left(Q_{X}^{l}\right)^{3}-6\left(Q_{X}^{q}\right)^{3}=0,}
\end{gathered}
$$

where $l, q, e, u, d$ stand, respectively, for the lepton and quark doublets, right-handed charged leptons, and the up-type and down-type right-handed quarks. $Q_{X_{2}}$ is, instead, the charge of the $\Phi_{2}$ field. In Appendix A, we describe how to find the above relations. By using them, it is possible to find several $U(1)_{X}$ anomaly free models [49], including the $B-L$ approached here.

Taking $Q_{X}^{u}$ and $Q_{X}^{d}$ as free parameters, the anomaly conditions are satisfied if the SM spectrum is augmented with three right-handed neutrinos with charge $Q_{X}^{n}=$ $-\left(Q_{X}^{u}+2 Q_{X}^{d}\right)$. Using the Yukawa Lagrangian in Eq.(2.1), we obtain $Q_{X}^{d}-Q_{X}^{q}+Q_{X_{2}}=$ $0, Q_{X}^{u}-Q_{X}^{q}-Q_{X_{2}}=0, Q_{X}^{e}-Q_{X}^{l}+Q_{X_{2}}=0$, which, combined with the anomaly conditions, allow us to express all the charges as function of $Q_{X}^{u}$ and $Q_{X}^{d}$ :

$$
\begin{array}{r}
Q_{X}^{q}=\frac{\left(Q_{X}^{u}+Q_{X}^{d}\right)}{2}, \\
Q_{X}^{l}=-\frac{3\left(Q_{X}^{u}+Q_{X}^{d}\right)}{2}, \\
Q_{X}^{e}=-\left(2 Q_{X}^{u}+Q_{X}^{d}\right), \\
Q_{X_{2}}=\frac{\left(Q_{X}^{u}-Q_{X}^{d}\right)}{2} .
\end{array}
$$

In Appendix A, we describe how to find the conditions for anomaly freedom. By using them, it is possible to find several $U(1)_{X}$ anomaly free models [49], as already mentioned above.

\subsection{Scalar Potential}

The scalar potential in the presence of two scalar doublets, transforming differently under $U(1)_{X}$, and a scalar singlet reads:

$$
\begin{aligned}
V\left(\Phi_{1}, \Phi_{2}\right) & =m_{11}^{2} \Phi_{1}^{\dagger} \Phi_{1}+m_{22}^{2} \Phi_{2}^{\dagger} \Phi_{2}+m_{s}^{2} \Phi_{s}^{\dagger} \Phi_{s}+\frac{\lambda_{1}}{2}\left(\Phi_{1}^{\dagger} \Phi_{1}\right)^{2}+\frac{\lambda_{2}}{2}\left(\Phi_{2}^{\dagger} \Phi_{2}\right)^{2}+ \\
& +\lambda_{3}\left(\Phi_{1}^{\dagger} \Phi_{1}\right)\left(\Phi_{2}^{\dagger} \Phi_{2}\right)+\lambda_{4}\left(\Phi_{1}^{\dagger} \Phi_{2}\right)\left(\Phi_{2}^{\dagger} \Phi_{1}\right)+ \\
& +\frac{\lambda_{s}}{2}\left(\Phi_{s}^{\dagger} \Phi_{s}\right)^{2}+\mu_{1} \Phi_{1}^{\dagger} \Phi_{1} \Phi_{s}^{\dagger} \Phi_{s}+\mu_{2} \Phi_{2}^{\dagger} \Phi_{2} \Phi_{s}^{\dagger} \Phi_{s}+\left(\mu \Phi_{1}^{\dagger} \Phi_{2} \Phi_{s}+\text { h.c. }\right) .
\end{aligned}
$$

Notice that, given the fact that the two Higgs doublets have different charged under the $U(1)_{X}$ group, only the tri-scalar operator $\Phi_{1}^{\dagger} \Phi_{2} \Phi_{s}+$ h.c. is allowed by the symmetries of the system. 
2HDM with right-handed neutrino dark matter

\begin{tabular}{ccccccc}
\hline Fields & $u_{R}$ & $d_{R}$ & $Q_{L}$ & $L_{L}$ & $e_{R}$ & $N_{R}$ \\
\hline$U(1)_{X}$ & $Q_{X}^{u}$ & $Q_{X}^{d}$ & $\frac{\left(Q_{X}^{u}+Q_{X}^{d}\right)}{2}$ & $\frac{-3\left(Q_{X}^{u}+Q_{X}^{d}\right)}{2}$ & $-\left(2 Q_{X}^{u}+Q_{X}^{d}\right)$ & $-\left(Q_{X}^{u}+2 Q_{X}^{d}\right)$ \\
$U(1)_{B-L}$ & $1 / 3$ & $1 / 3$ & $1 / 3$ & -1 & -1 & -1 \\
\hline & & & Fields & $\Phi_{2}$ & $\Phi_{1}$ & \\
\cline { 4 - 6 } & & & $U(1)_{X}$ & $\frac{\left(Q_{X}^{u}-Q_{X}^{d}\right)}{2}$ & $\frac{5 Q_{X}^{u}}{2}+\frac{7 Q_{X}^{d}}{2}$ & \\
& & & $U(1)_{B-L}$ & 0 & 2 \\
\hline
\end{tabular}

Table 1. Field context of our $2 \mathrm{HDM}-U(1)_{X}$ model where the lightest right-handed neutrino is dark matter, whose stability is protected by a $Z_{2}$ symmetry.

The two doublets and the singlet can be decomposed as:

$$
\begin{gathered}
\Phi_{i}=\left(\begin{array}{c}
\phi_{i}^{+} \\
\left(v_{i}+\rho_{i}+i \eta_{i}\right) / \sqrt{2}
\end{array}\right), \\
\Phi_{s}=\frac{1}{\sqrt{2}}\left(v_{s}+\rho_{s}+i \eta_{s}\right) .
\end{gathered}
$$

From the terms of Eqs.(2.2)-(2.10) we can determine the following relations: $Q_{X_{s}}=$ $Q_{X_{1}}-Q_{X_{2}}$ and $-Q_{X}^{l}-Q_{X}^{e}+Q_{X}^{n}=0$, leading to $Q_{X_{1}}=\frac{5 Q_{X}^{u}}{2}+\frac{7 Q_{X}^{d}}{2}$. The values of the $U(1)_{X}$ charges, both in the general case and for $X=B-L$, the specific case of study of this work, for the field content of our model are summarized in Table 1.

After EW symmetry breaking and assuming that $\mathrm{CP}$ is preserved by the scalar potential, the CP-even and CP-odd components of the Higgs fields will mix, eventually leading to three CP-even mass eigenstates, which we indicate with the standard notation $h, H$ and $h_{s}$, two charged states $H^{ \pm}$and one CP-odd state, $A$. Throughout this study, we will identify $h$ with the $125 \mathrm{GeV}$ SM-like Higgs and $h_{s}$ with a mostly singlet-like Higgs, with negligible mixing with the other CP-even scalars. In such a case the mass of the latter is simply given by:

$$
m_{h_{s}}^{2} \simeq \lambda_{s} v_{s}
$$

The other relevant parameter for DM phenomenology is the mass of the charged Higgs, given by $[49,85]$ :

$$
m_{H^{ \pm}}^{2} \simeq \frac{\left(\sqrt{2} \mu v_{s}-\lambda_{4} v_{1} v_{2}\right) v^{2}}{2 v_{1} v_{2}}
$$

In order to ensure negligible mixing between the Higgs doublets and singlet the $\mu$ parameter should be small but still satisfy:

$$
\mu>\frac{\lambda_{4} v_{1} v_{2}}{\sqrt{2} v_{s}}
$$

Unless differently stated, we will assume, for our analysis, the assignations $\lambda_{4}=0.1$, $\lambda_{s}=0.1$ and $\mu=35 \mathrm{GeV}$. 


\subsection{Relevant Interactions}

Let's start with the interactions of the gauge bosons. The new $U(1)_{X}$ and the hypercharge gauge boson are described by the following Lagrangian:

$$
\mathcal{L}_{\text {gauge }}=-\frac{1}{4} \hat{B}_{\mu \nu} \hat{B}^{\mu \nu}+\frac{\epsilon}{2 \cos \theta_{W}} \hat{X}_{\mu \nu} \hat{B}^{\mu \nu}-\frac{1}{4} \hat{X}_{\mu \nu} \hat{X}^{\mu \nu} .
$$

As we added a $U(1)_{X}$ gauge group a kinetic mixing between the two Abelian groups is, in general, allowed by the gauge symmetries. Assuming it to be zero at the tree level, we can nevertheless not avoid its generation at the one-loop level since the SM fermion are charged under both the two gauge group. In such case the induced kinetic mixing parameter $\epsilon$ can be estimated as $|\epsilon| \sim g^{\prime} g_{X} /\left(16 \pi^{2}\right) \sum Q_{X}^{2} \ln M^{2} / \mu^{2}$ $[91,92]$ where, again, $g_{X}$ is the gauge coupling of the $U(1)_{X}$ and $Q_{X}$ is the charge of the SM fermion. This expression roughly gives $\epsilon \sim 10^{-2} g_{X}$; consequently we can assume that kinetic mixing gives a subdominant contribution to DM phenomenology and, hence, neglect it in numerical computation.

The Lagrangian responsible for the mass of the gauge bosons is:

$$
\begin{aligned}
& \mathcal{L}=\left(D^{\mu} \phi_{1}\right)^{\dagger}\left(D_{\mu} \phi_{1}\right)+\left(D^{\mu} \phi_{2}\right)^{\dagger}\left(D_{\mu} \phi_{2}\right)+\left(D^{\mu} \phi_{s}\right)^{\dagger}\left(D_{\mu} \phi_{s}\right)= \\
& +\frac{1}{4} g^{2} v^{2} W^{-\mu} W_{\mu}^{+}+\frac{1}{8} g_{Z}^{2} v^{2} Z^{0 \mu} Z_{\mu}^{0}-\frac{1}{4} g_{Z}\left(G_{X_{1}} v_{1}^{2}+G_{X_{2}} v_{2}^{2}\right) Z^{0 \mu} X_{\mu} \\
& +\frac{1}{8}\left(v_{1}^{2} G_{X_{1}}^{2}+v_{2}^{2} G_{X_{2}}^{2} v_{2}^{2}+v_{s}^{2} Q_{X_{s}}^{2} g_{X}^{2}\right) X^{\mu} X_{\mu}
\end{aligned}
$$

where we have used the following expression for the covariant derivative:

$$
D_{\mu}=\partial_{\mu}+i g T^{a} W_{\mu}^{a}+i g^{\prime} \frac{Q_{Y}}{2} \hat{B}_{\mu}+i g_{X} \frac{Q_{X}}{2} \hat{X}_{\mu}
$$

while:

$$
G_{X_{i}}=\frac{\epsilon Q_{Y_{i}}}{\cos \theta_{W}}+g_{X} Q_{X_{i}}
$$

From the equation above we straightforwardly recover the SM value for the mass of the $\mathrm{W}$ boson:

$$
m_{W}^{2}=\frac{1}{4} g^{2} v^{2}
$$

the masses of the remaining gauge bosons are obtained upon diagonalization of the following mass matrix:

$$
\mathcal{M}=\left(\begin{array}{cc}
g_{Z}^{2} v^{2} & -g_{Z}\left(G_{X_{1}} v_{1}^{2}+G_{X_{2}} v_{2}^{2}\right) \\
-g_{Z}\left(G_{X_{1}} v_{1}^{2}+G_{X_{2}} v_{2}^{2}\right) & v_{1}^{2} G_{X_{1}}^{2}+v_{2} G_{X_{2}}^{2} v_{2}^{2}+q_{X}^{2} g_{X}^{2} v_{s}^{2}
\end{array}\right)
$$

which leads to the following rotation:

$$
\left(\begin{array}{l}
Z^{\mu} \\
Z^{\prime \mu}
\end{array}\right)=\left(\begin{array}{cc}
\cos \xi & -\sin \xi \\
\sin \xi & \cos \xi
\end{array}\right)\left(\begin{array}{c}
Z^{0 \mu} \\
X^{\mu}
\end{array}\right)
$$


where the mixing $\xi$ is defined in general as

$$
\tan 2 \xi=\frac{2 g_{Z}\left(G_{X_{1}} v_{1}^{2}+G_{X_{2}} v_{2}^{2}\right)}{m_{Z_{0}}^{2}-m_{X}^{2}}
$$

However, in this work we will mostly consider the regime $m_{X}^{2} \gg m_{Z^{0}}^{2},{ }^{3}$ (we will further comment on this choice in the next section) and very small mixing angle. In such a case we can write

$$
\begin{aligned}
& m_{Z}^{2} \simeq m_{Z^{0}}^{2}=\frac{1}{4} g_{Z}^{2} v^{2} \\
& m_{Z^{\prime}}^{2} \simeq m_{X}^{2}=\frac{v_{s}^{2}}{4} g_{X}^{2} Q_{X_{s}}^{2}+\frac{g_{X}^{2} v^{2} \cos ^{2} \beta \sin ^{2} \beta}{4}\left(Q_{X_{1}}-Q_{X_{2}}\right)^{2}
\end{aligned}
$$

and

$$
\begin{aligned}
& \sin \xi \simeq \frac{G_{X_{1}} v_{1}^{2}+G_{X_{2}} v_{2}^{2}}{m_{Z^{\prime}}^{2}} \\
& =\frac{m_{Z}^{2}}{m_{Z^{\prime}}^{2}}\left(\frac{g_{X}}{g_{Z}}\left(Q_{X_{1}} \cos ^{2} \beta+Q_{X_{2}} \sin ^{2} \beta\right)+\epsilon \tan \theta_{W}\right) .
\end{aligned}
$$

Finally, we write the Lagrangian describing the neutral current interactions as follows:

$$
\begin{aligned}
\mathcal{L}_{\mathrm{NC}}= & -e J_{\mathrm{em}}^{\mu} A_{\mu}-\frac{g}{2 \cos \theta_{W}} \cos \xi J_{N C}^{\mu} Z_{\mu}-\sin \xi\left(\epsilon e J_{e m}^{\mu}+\epsilon_{Z} \frac{g}{2 \cos \theta_{W}} J_{N C}^{\mu}\right) Z_{\mu}^{\prime}+ \\
& +\frac{1}{4} g_{X} \sin \xi\left[\left(Q_{X f}^{R}+Q_{X f}^{L}\right) \bar{\psi}_{f} \gamma^{\mu} \psi_{f}+\left(Q_{X f}^{R}-Q_{X f}^{L}\right) \bar{\psi}_{f} \gamma^{\mu} \gamma_{5} \psi_{f}\right] Z_{\mu}+ \\
& -\frac{1}{4} g_{X} \cos \xi\left[\left(Q_{X f}^{R}+Q_{X f}^{L}\right) \bar{\psi}_{f} \gamma^{\mu} \psi_{f}-\left(Q_{X f}^{L}-Q_{X f}^{R}\right) \bar{\psi}_{f} \gamma^{\mu} \gamma_{5} \psi_{f}\right] Z_{\mu}^{\prime}+ \\
& -\frac{1}{4} Q_{N_{1}} g_{X} \cos \xi \cos \xi N_{1} \gamma^{\mu} \gamma_{5} N_{1} Z_{\mu}^{\prime}+\frac{1}{4} Q_{N_{1}} g_{X} \sin \xi N_{1} \gamma^{\mu} \gamma_{5} N_{1} Z_{\mu}
\end{aligned}
$$

where we have again adopted a general notation in terms of generic charges for the DM and the SM fermions under the new gauge symmetry (conditions for an anomaly free symmetry are, of course, automatically assumed).

Besides the neutral current there are other interactions that might be relevant for our phenomenology such $Z^{\prime} W^{+} W^{-}, Z^{\prime} W^{+} H^{-}, H Z^{\prime} Z^{\prime}, h Z^{\prime} Z^{\prime}, H Z Z^{\prime}$. The presence of such interactions is an additional feature that distinguishes our work from previous studies in the literature $[88,94,95]$. In particular, the $Z^{\prime} W^{+} H^{-}$is proportional to $g g_{X} v / 2 \cos \xi$, therefore it cannot be neglected. The details expression for the aforementioned couplings can be found e.g. in [85].

\footnotetext{
[93].

${ }^{3}$ In the opposite regime the same model can be used to interpret the recent XENON1T anomaly
} 


\section{Dark Matter Phenomenology}

\subsection{Relic Density - Standard Cosmology}

Assuming a standard cosmological history the DM relic density can be determined through the thermal freeze-out paradigm, so that:

$$
\Omega_{N_{1}} h^{2} \propto \frac{1}{\langle\sigma v\rangle} .
$$

The thermally favored value of the DM relic density $\Omega_{N_{1}} h^{2} \approx 0.12$ [1] is achieved when the thermally averaged DM pair annihilation cross-section is of the order of $10^{-26} \mathrm{~cm}^{3} \mathrm{~s}^{-1}$. The main contributions to the DM annihilation cross-section come from pair annihilation into SM fermions pairs, determined by the interactions written in Eq. (2.25), as well as, if kinematically allowed, annihilation into gauge boson pairs and Higgs/gauge boson final states. In the regime $\sin \xi \ll 1$ the most relevant final states are $Z^{\prime} Z^{\prime}$ and $W^{ \pm} H^{\mp}$ and $Z^{\prime} h_{s}$. In order to properly account for this broad variety of annihilation channels we have implemented the $2 H D M+U(1)_{B-L}$ model into the package micrOMEGAs $[96,97]$ and we have numerically determined the DM relic density as function of the DM and Z' masses, $m_{Z^{\prime}}, m_{N_{1}}$, and of the gauge coupling $g_{X}$.

\subsection{Relic Density - Early Matter Domination}

As will be evidenced in the following, the correct relic density can be achieved, according to conventional thermal production and compatibly with the experimental constraints, which will be illustrated below, only in very narrow regions of the parameter space. We will then explore the possibility that the viable parameter space for the model is enlarged in the case of non-standard cosmological history for the Early Universe, due to the presence of a scalar field $\phi$, dominating the energy budget of the Universe for some amount of time prior to its decay.

The most general strategy to deal with this non-standard scenario consists into solving the following system of Boltzmann equations [98-104]:

$$
\begin{array}{r}
\frac{d \rho_{\phi}}{d t}+3(1+\omega) H \rho_{\phi}=\Gamma_{\phi} \rho_{\phi}, \\
\frac{d s}{d t}+3 H s=\frac{\Gamma_{\phi} \rho_{\phi}}{T}\left(1-2 \frac{E}{m_{\phi}} B R_{N}\right)+2 \frac{E}{T}\langle\sigma v\rangle\left(n_{N}^{2}-n_{N e q}^{2}\right), \\
\frac{d n_{N}}{d t}+3 H n_{N}=\frac{2}{m_{\phi}} \Gamma_{\phi} \rho_{\phi} B R_{N}-\langle\sigma v\rangle\left(n_{N}^{2}-n_{N e q}^{2}\right),
\end{array}
$$

tracking the time evolution of, respectively, the energy density of the scalar field, the entropy density of the primordial plasma and the DM number density. The averaged energy per dark matter particle as a function of the temperature $T$ is $E^{2} \simeq m_{N}^{2}+3 T^{2}$ while $m_{\phi}$ and $\Gamma_{\phi}$ are, respectively, the mass and decay width of the scalar $\phi$, and 
$\omega=p_{\phi} / \rho_{\phi}$ is the equation-of-state parameter of the field $\phi$. We will consider the scenario of an Early matter domination, hence we set $\omega=0$. We will further assume that the new scalar field cannot decay into DM pairs, i.e. $B R_{N}=0$, for example because its charge under the $B-L$ symmetry is $\neq-2$.

In such a case, the main effect on the DM relic density is a strong dilution of the thermally produced component of the DM, due to the entropy injection following $\phi$ decay. This dilution should be compensated through a comparable overabundance, with respect to the thermally favored values, of the thermally produced DM. The latter is achieved by requiring smaller values, with respect to the standard freeze-out case, for the $g_{X}$ coupling, so that the annihilation cross-section of the DM is suitably suppressed. As can be easily argued this will have, in turn, profound implications in the complementarity between relic density and experimental searches.

Given the simplifying assumptions illustrated above, a good approximation of the impact from the aforementioned dilution process on the DM relic density can be obtained in the following way. First of all, let's define the temperature at which the early matter domination phase ends. It is simply obtained by imposing:

$$
\Gamma_{\phi}=H\left(T_{\text {end }}\right)=\sqrt{\frac{\pi^{2}}{90} g_{*}\left(T_{\text {end }}\right)} T_{\text {end }}=\left(\frac{90}{\pi^{2} g_{*}\left(T_{\text {end }}\right)} M_{P l}^{2} \Gamma_{\phi}^{2}\right)^{1 / 4},
$$

where $\Gamma_{\phi}$ is the decay rate of the scalar field. The second important parameter is the ratio between the energy density of the scalar field over the one stored into radiation, computed at temperature of the order of the DM mass:

$$
\kappa=\left.\frac{\rho_{\phi}}{\rho_{R}}\right|_{T=m_{N}} .
$$

In the regime $T_{\text {eq }} \ll T_{\text {f.o. }}$, where $T_{\text {eq }}$ and $T_{\text {f.o. }}$ are, respectively, the temperature at which $\rho_{\phi}>\rho_{R}$ and the standard freeze-out temperature, the DM relic density can be simply computed as:

$$
\Omega_{N} h^{2}=\frac{\Omega_{N}^{\text {s.f.o. }} h^{2}}{D}
$$

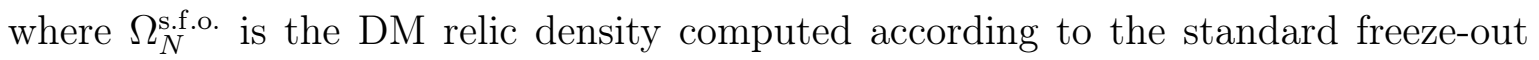
paradigm while $D$ is the dilution factor defined as:

$$
D=\frac{s\left(T_{2}\right)}{s\left(T_{1}\right)}
$$

i.e. the ratio of the entropy density at temperatures immediately after and before the decay of the scalar field. The dilution factor can be straightforwardly computed in the instantaneous decay approximation. In such a case, the conservation of energy implies that:

$$
\rho_{\phi}\left(T_{1}\right)+\rho_{R}\left(T_{1}\right)=\rho_{R}\left(T_{2}\right),
$$

using $\rho_{\phi}\left(m_{N}\right)=\kappa \rho_{R}\left(m_{N}\right)$ and setting $T_{2}=T_{\text {end }}$ the dilution factor is given by [103]: 


$$
D=\kappa \frac{m_{N}}{T_{e n d}}
$$

The range of values of $D$ is limited by the fact that $T_{\text {end }}$ cannot fall below around $5 \mathrm{MeV}$ due to Big Bang Nucleosynthesis contraints [105-108]. Moreover the ratio between $\rho_{\phi}$ and $\rho_{R}$ should become greater than one only at temperatures well below the one of standard freeze-out of the DM, since the solution illustrated above is valid only in this regime. Connected to this we notice that the dependence of $D$ on the DM mass is only apparent since $\rho_{\phi} / \rho_{R} \propto 1 / T \rightarrow \kappa \propto m_{N}$. Having this in mind we have considered, for our analysis, two specific values of $D$, namely 550 and 2750, assuming in both cases $T_{\text {end }}=7 \times 10^{-3} \mathrm{GeV}$.

\subsection{Direct Detection}

Direct detection refers to the measurement of nuclear recoils caused by dark matter scatterings off nuclei. As the momentum transfer is small compared to the mass of the mediator that controls the scattering we can apply effective field theory. In the limit $\sin \xi \rightarrow 0$ the relevant Lagrangian is:

$$
\mathcal{L}_{D D}=\frac{g_{X}^{4}}{18 m_{Z^{\prime}}^{2}} \bar{N} \gamma^{\mu} \gamma_{5} N \bar{q} \gamma_{\mu} q
$$

where $q=u, d$. The interactions with electrons have been neglected from Eq. (3.11) as they are important when we are dealing with light dark matter. The Lagrangian (3.11) does not lead to the conventional spin-independent (SI) or spin-dependent (SD) interactions.

To determine eventual constraints we have to rely on the more general formalism, as introduced e.g in [109-111]. According to this, the effective Lagrangian (3.11) can be mapped into the following non-relativistic operators [111-113]:

$$
\bar{N} \gamma^{\mu} \gamma_{5} N \bar{q} \gamma_{\mu} q \rightarrow 2 \vec{v}_{N}^{\perp} \cdot \vec{S}_{N}+2 i \vec{S}_{N} \cdot\left(\vec{S}_{n} \times \frac{\vec{q}}{m_{N}}\right)
$$

where, $\vec{S}_{N, n}$ are the DM and nucleus spins, $\vec{v}_{N}^{\perp}=\vec{v}+\frac{\vec{q}}{2 \mu_{N n}}$ and $\vec{q}$ is the momentum transfer. As the first term does not depend on the nucleus's spin, it experiences the same $A^{2}$ enhancement typical of spin-independent interactions, with $\mathrm{A}$ being the atomic mass of the nucleus. For instance, for ${ }^{131} \mathrm{Xe}$ this term is boosted by $\sim 1.7 \times 10^{4}$. However, one should keep in mind that it is also velocity suppressed. As this operator yields a distinct recoil spectrum we cannot directly apply the experimental limits on the scattering cross section. In order to compute the direct detection bounds on the parameter space of interest we used the code described in [112]. The limit can be found by computing the differential recoil rate in a xenon detector after considering efficiency and no background events. We point out that the renormalization group effects studied in [114] are not important here as we have a vector quark current.

We have compared the expected DM scattering rate with the limits given by the XENON1T experiment and found that they can constraint the viable DM parameter 
space only for $g_{X}>1$. As will be evidenced by Fig. 1 , for $g_{X}=1$, the $Z / Z^{\prime}$ cannot be neglected. The latter generates the $\bar{N}_{1} \gamma^{\mu} \gamma^{5} N_{1} \bar{q} \gamma_{\mu} \gamma_{5} q$ operator, responsible of the conventional SD interaction, with a cross-section given by:

$$
\sigma_{\mathrm{N}_{1 \mathrm{p}}}^{\mathrm{SD}}=\frac{\mu_{N_{1} p}^{2}}{4 \pi}\left|\left(\frac{g_{Z u}^{A} g_{Z N_{1}}}{m_{Z}^{2}}+\frac{g_{Z^{\prime} u}^{A} g_{Z^{\prime} N_{1}}}{m_{Z^{\prime}}^{2}}\right) \Delta_{u}^{p}+\left(\frac{g_{Z d}^{A} g_{Z N_{1}}}{m_{Z}^{2}}+\frac{g_{Z^{\prime} d}^{A} g_{Z^{\prime} N_{1}}}{m_{Z^{\prime}}^{2}}\right)\left(\Delta_{d}^{p}+\Delta_{s}^{p}\right)\right|^{2}
$$

where $\Delta_{u, d, s}^{p}$ are form factors representing the contribution of light quarks to the nucleon spin. $g_{Z\left(Z^{\prime}\right) u, d}^{A}$ are the axial couplings of the $Z, Z^{\prime}$ with up-type and down-type quarks while $g_{Z\left(Z^{\prime}\right) N_{1}}$ are the couplings of the neutral gauge bosons with the DM. Their explicit expressions can be straightforwardly inferred from Eq. (2.25).

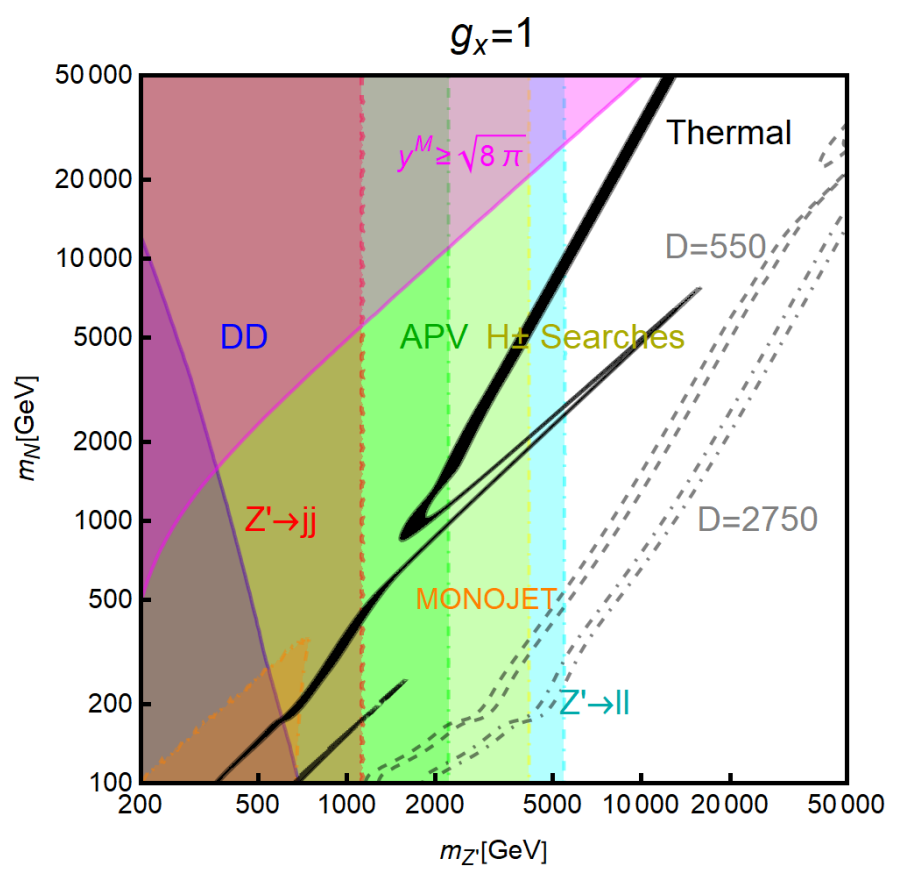

Figure 1. Region of parameter space in the right-handed neutrino versus $Z^{\prime}$ mass plane that yields the correct relic density (solid line) overlaid with existing bounds from direct detection, and collider searches, for $g_{\chi}=1$. Moreover, we have added the curves that delimit the region of parameter space that yields the correct relic density in the presence of an early matter domination episode occurring after the dark matter freeze-out. See text for details.

\subsection{Indirect Detection}

The dark matter annihilation cross section into fermions is velocity suppressed (i.e p-wave like ${ }^{4}$, useful related analytical expressions can be found e.g. in $[2,115]$ ) so it is not responsible for significant and detectable indirect signals. The annihilation into $Z^{\prime}$ pairs is not velocity suppressed but is subdominant unless $0.5 m_{N}<m_{Z^{\prime}}<0.9 m_{N}$.

\footnotetext{
${ }^{4} \mathrm{An}$ s-wave contribution is actually present but it is helicity suppressed. It is then, in general, subdominant
} 
As we are considering $m_{Z^{\prime}} \geq 200 \mathrm{GeV}$, such annihilation processes are relevant for $m_{N_{1}}>200 \mathrm{GeV}$. Indirect Detection constraints and Cosmic Microwave Background radiation searches cannot yet probe the thermally favored value of the DM annihilation cross-section for these high values of the DM mass [116, 117]. Furthermore, collider constraints are much more competitive, excluding $Z^{\prime}$ masses up to few $\mathrm{TeV}$. Since similar arguments hold also for the other s-wave dominated annihilation final states, i.e. the one into $Z^{\prime} h_{s}$, we conclude that indirect detection does not play a significant role in our study.

\subsection{Perturbative Unitarity}

One can use perturbative unitarity to constrain sums of couplings that are relevant for our phenomenology. Considering self-interactions, $N N \rightarrow N N$, we can roughly impose $y_{a b}^{M}<\sqrt{8 \pi}$. As the right-handed neutrino mass and the $Z^{\prime}$ mass are governed by the same vev $\left(v_{s}\right)$ we can use this relation to impose $g_{\chi} m_{\chi} / m_{Z^{\prime}}<\sqrt{\pi}[113,118]$. The region of the parameter space where this condition is not satisfied is shaded in magenta.

\subsection{Collider bounds}

It is well know that new gauge boson can be constrained by a broad variety of collider searches. Thanks to their gauge interactions with SM fermions, $Z^{\prime}$ bosons can be efficiently produced in proton-proton collisions. $Z^{\prime}$ visible decay products can be then searched for through searches of dijet and/or dilepton resonances. If kinematically allowed, $Z^{\prime}$ decays into DM pairs lead to the so-called mono-X events. Among different searches strategies, dileptons and dijet are normally the most effective in constraining the model $[2,119]$. While being in general less restrictive, mono-X searches can nevertheless provide useful complementary information.

For our analysis we have adopted the following bounds:

- Searches for heavy $\left(m_{Z^{\prime}} \gtrsim 1 \mathrm{TeV}\right)$ dijet resonances from ATLAS [120];

- Searches for light dijet resonances from ATLAS [121] and CMS [122], the latter being sensitive to masses of the $Z^{\prime}$ down to $45 \mathrm{GeV}$;

- Searches of heavy $\left(m_{Z^{\prime}} \gtrsim 600 \mathrm{GeV}\right)$ dilepton resonances from ATLAS [123];

- Searches for resonances decaying into top-quark pairs by ATLAS [124]. Since the corresponding limit is not competitive with the ones coming from dijet and dilepton searches, it has not been shown explicitly shown in the figures;

- Searches for monojet events by ATLAS [125]. 


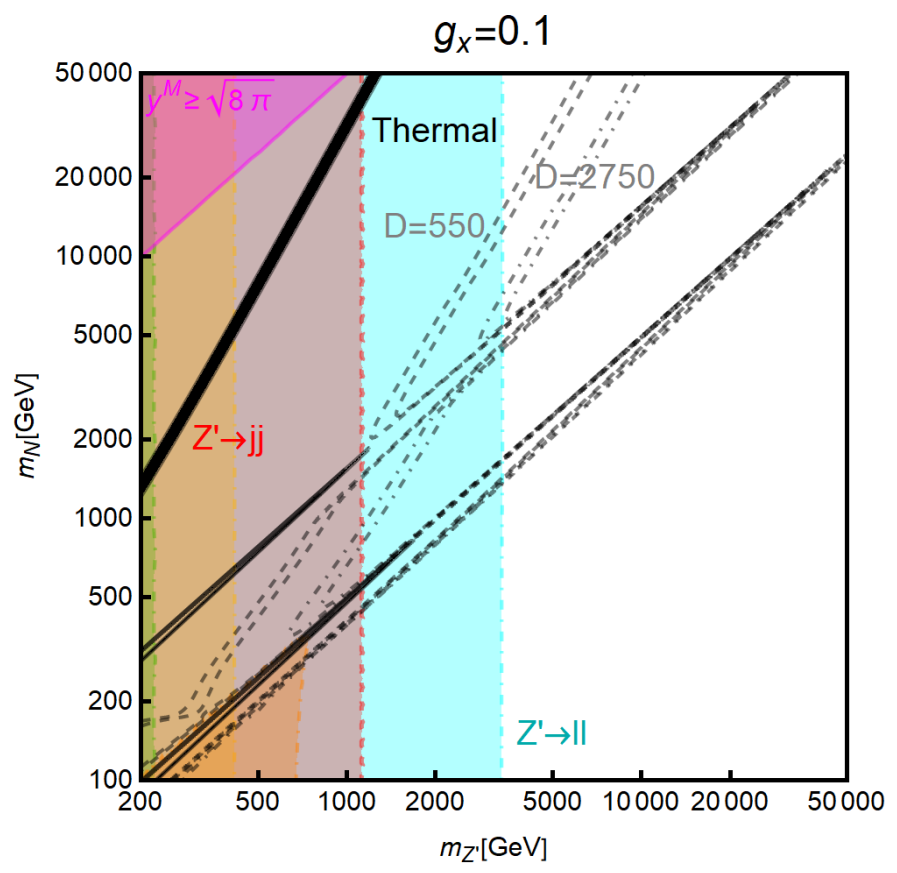

Figure 2. Same as Fig.1, but for $g_{\chi}=0.1$.

\subsection{Atomic Parity violation}

Atomic parity violation (APV) is a powerful complementary probe of new physics $[49,76]$. Since we are considering a heavy $Z^{\prime}$, APV effects can be described through the following effective Lagrangian:

$$
\mathcal{L}_{\mathrm{APV}}=\left(\frac{g_{Z e}^{A} g_{Z u}^{V}}{m_{Z}^{2}}+\frac{g_{Z^{\prime} e}^{A} g_{Z^{\prime} u}^{V}}{m_{Z^{\prime}}^{2}}\right) \bar{e} \gamma^{\mu} \gamma_{5} e \bar{u} \gamma_{\mu} u+\left(\frac{g_{Z e}^{A} g_{Z d}^{V}}{m_{Z}^{2}}+\frac{g_{Z^{\prime} e}^{A} g_{Z^{\prime} d}^{V}}{m_{Z^{\prime}}^{2}}\right) \bar{e} \gamma^{\mu} \gamma_{5} e \bar{d} \gamma_{\mu} d
$$

where $g_{Z\left(Z^{\prime}\right) f}^{V, A}$ are the vector $(V 4)$ and axial $(A)$ couplings of the $Z\left(Z^{\prime}\right)$ gauge bosons with SM fermions. APV can be experimentally probed by looking for Left-Right asymmetries in atomic transitions. Experimental limits can be expressed in terms of constraints on the deviation $\Delta Q_{W}=Q_{W}-Q_{W}^{\mathrm{SM}}$ with $Q_{W}=Q_{W}(Z, N)$ being the weak charge of target nucleus while $Q_{W}^{\mathrm{SM}}$ is the SM prediction for the same quantity.

In the limit of small $Z / Z^{\prime}$ mixing angle we can write:

$$
\begin{aligned}
\Delta Q_{W} & =\left[-\delta^{2} Q_{W}^{\mathrm{SM}}-4 \delta^{2} Z \sin \theta_{W} \cos \theta_{W} \frac{\epsilon}{\epsilon_{Z}}-(2 Z+N) \delta^{2} \frac{\left(Q_{X}^{u}+Q_{X}^{q}\right)}{Q_{X_{1}} \cos ^{2} \beta+Q_{X_{2}} \sin ^{2} \beta}+\right. \\
& \left.-(Z+2 N) \delta^{2} \frac{\left(Q_{X}^{q}+Q_{X}^{d}\right)}{Q_{X_{1}} \cos ^{2} \beta+Q_{X_{2}} \sin ^{2} \beta}\right]\left(1-\frac{Q_{X}^{l}-Q_{X}^{e}}{Q_{X_{1}} \cos ^{2} \beta+Q_{X_{2}} \sin ^{2} \beta}\right)
\end{aligned}
$$

where [49]:

$$
\delta=\frac{2 \cos \beta \cos \beta_{s}}{\sqrt{Q_{X_{s}}^{2}+\cos ^{2} \beta_{s}\left(\sin ^{2} \beta\left(Q_{X_{1}}-Q_{X_{2}}\right)^{2}-q_{X}^{2}\right)}}
$$

and where we have defined $\tan \beta_{s}=\frac{v_{1}}{v_{s}}$. 
At the moment, the strongest constraints from APV come from Cesium transitions [126]. Substituting $Z=55, N=78$ and the central value of the $\mathrm{SM}$ expectation $Q_{W}^{\mathrm{SM}}=-73.16$ and by specializing to the $B-L$ model, we find:

$$
\Delta Q_{W}=-59.84 \delta^{2}-220 \delta \sin \theta_{W} \cos \theta_{W} \epsilon \frac{m_{Z}}{m_{Z^{\prime}}}-133 \delta^{2} \tan \beta^{2} .
$$

Imposing the bound [76]:

$$
\left|\Delta Q_{W}\right|<0.6
$$

one can obtain a lower bound, as function of the coupling $g_{X}$, on the mass of the $Z^{\prime}$ which can possibly complement the ones from collider searches of $Z^{\prime}$.

\subsection{Constraints on extra scalars}

Albeit DM observables can feature or not a dependence on the masses of the additional Higgs bosons besides the SM-like Higgs $h$, these additional scalars can impact the parameter space under consideration, since they are related, through the gauge coupling

$g_{X}$ and the singlet $v e v v_{s}$, to the mass of the $Z^{\prime}$. In the scenario we consider here, the strongest constraints come from collider searches for the charged Higgs boson $H^{ \pm}$as well as from effects on $b \rightarrow s$ transitions. A recent review of these constraints can be found in [127].

\section{Results}

We present our results in the dark matter vs mediator mass plane, for $g_{X}=1,0.1,0.01$. The black lines and shaded regions correspond to parameter space that provides the "right" relic density assuming a standard cosmological history. The dashed (dot-dashed) gray contours represent instead the correct relic density in the case of an epoch of early matter domination after DM freeze-out ending with the decay of a scalar field with dilution of the thermally generated DM abundance by a factor $D=550(2750)$. In both cases, the epoch of matter domination ends at a temperature $T_{\text {end }}=7 \mathrm{MeV}$.

As anticipated, the case $g_{X}=1$ is extremely constrained, with the strongest bound from collider searches for dilepton resonances ruling out masses of the $Z^{\prime}$ up to $5 \mathrm{TeV}$. Assuming the standard freeze-out scenario, a viable parameter space is achieved only in correspondence with the $m_{N} \sim \frac{m_{Z^{\prime}}}{2}$ "pole" and, in a rather small window, in the $m_{N}>m_{Z^{\prime}}$ regime, between the region excluded by dilepton searches and by the unitarity bound. In all cases, DM masses in the multi-TeV range are required. Notice that the relic density contour levels in Fig. 1 show a second small cusp: This

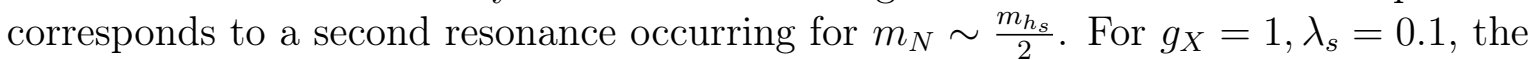
$h_{S}$ is lighter than the $Z^{\prime}$. For this choice of parameters, resonant enhancement from $s$-channel exchange of the $h_{s}$ would occur mainly through the $N_{1} N_{1} \rightarrow Z Z$ process, whose rate is suppressed as $\sin \xi^{4}$; for this reason, the second cusp is barely visible in the plot. Furthermore it lies in a region excluded by experimental constraints.

The picture substantially changes when a non-standard cosmological history is considered. In such a case, a viable DM thermal relic density can be achieved for 
$m_{N}<m_{Z^{\prime}}$ away from resonances, with the DM as light as around $300 \mathrm{GeV}$. The case $g_{X}=0.1$ appears to be more constrained. This is due to the fact that the DM annihilation cross-section decreases as $g_{X}^{4}$, while the production cross-section for resonances only scales as $g_{X}^{2}$. Furthermore, as $g_{X}$ decreases, the extra Higgs bosons becomes increasingly heavier with respect to $m_{Z^{\prime}}$, thus reducing the kinematicallyaccessible final states.

A standard WIMP scenario appears to be completely ruled out, while values of $m_{N}$ greater than $5(1) \mathrm{TeV}$ become viable in the $D=550(2750)$ case. Contrary to the $g_{X}=1$ case (see Fig. 2), the thermal relic density contours feature two equally pronounced cusps. Indeed, for $g_{X}=0.1, m_{h_{S}}>m_{Z^{\prime}}$ with a resulting $s$-channel enhancement of the $N_{1} N_{1} \rightarrow Z^{\prime} Z^{\prime}$ process, whose rate is not suppressed by the small $Z / Z^{\prime}$ mixing angle.

Finally, in the $g_{X}=0.01$ case, Fig. 3 , the assumption of an early matter domination epoch is the only option to achieve the correct DM relic density for DM masses as low as $150 \mathrm{GeV}$, albeit at the price of a sizable fine-tuning: the $s$-channel resonance enhancement must be compounded with the entropy dilution effect from the decay of the scalar field $\phi$.

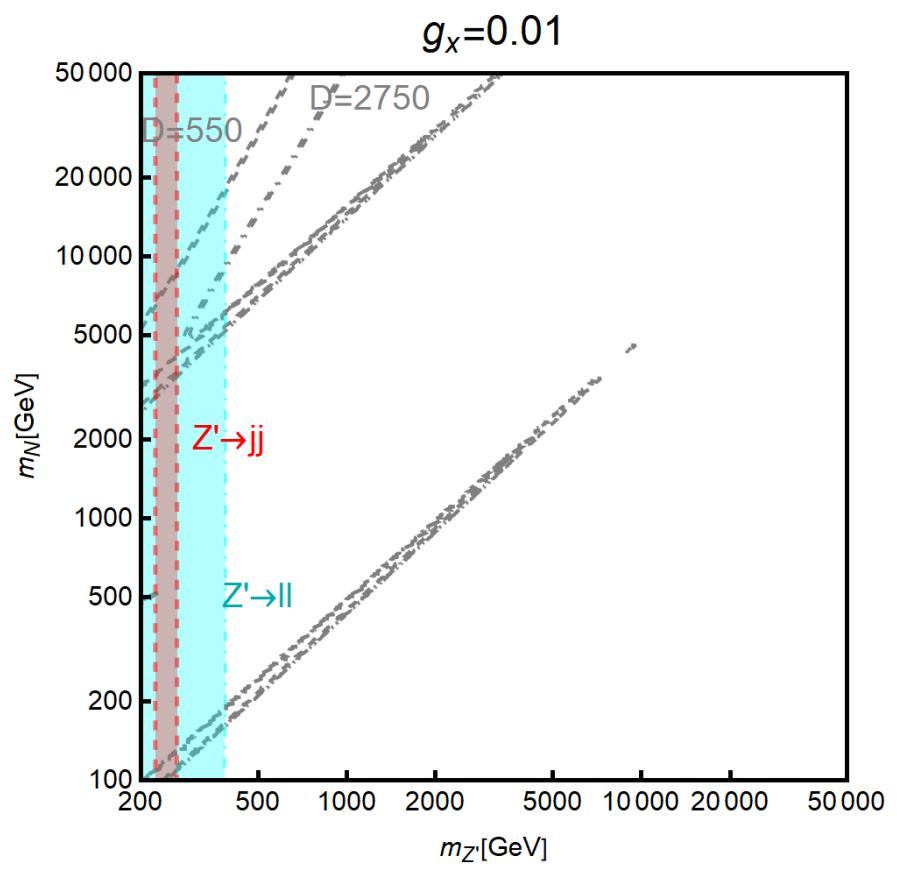

Figure 3. Same as Fig.1, but for $g_{\chi}=0.01$

As mentioned, we focused on the $m_{Z^{\prime}}>m_{Z}$ case. Viable DM relic density can also be obtained, through the mechanisms considered above, for $m_{Z^{\prime}}<m_{Z}$ as well; However, for $10 \lesssim m_{Z^{\prime}} \lesssim m_{Z}$, the viable parameter space for the correct thermal relic density is completely ruled out by LHC searches for light resonances decaying into muon pairs [123]. In the case of $\mathrm{GeV}$ or sub-GeV masses for the $Z^{\prime}$, values of $g_{X}$ as low as $10^{-3}$ are excluded by APV, and by searches for light $Z^{\prime}$ from BaBar $[128,129]$ and neutrino-electron scattering experiments [130-135]. 


\section{Conclusions}

In this study we have discussed a 2HDM model augmented by a spontaneously broken Abelian gauge symmetry; the model solves the flavor problem of the general 2HDM, while at the same time accommodating observed neutrino masses and mixing via a type-I seesaw mechanism. One of the right-handed neutrino is stable, and is a viable weak-scale thermal dark matter candidate. The right-handed neutrino interacts with a $\mathrm{TeV}$ scale $Z^{\prime}$ gauge boson that arises from the spontaneously broken $U(1)_{X}$ gauge symmetry, with its thermal relic abundance from the usual freeze-out paradigm, with or without a non-standard cosmology featuring a period of early matter domination ending at a temperature around $7 \mathrm{MeV}$, and thus compatible with constraints from the synthesis of light elements. Since the right-handed neutrino is a Majorana particle, and the $Z^{\prime}$ boson only has vectorial currents with SM fermions, direct detection is different from the usual spin-independent and spin-dependent classification; this required a direct comparison with the scattering rate from e.g. the XENON experiment. After considering perturbative unitarity, dijet and dilepton bounds from the LHC, we showed which region of parameter space satisfies current constraints, and made concrete predictions for observable features of this scenarios at future experiments.

\section{Acknowledgements}

SP is partly supported by the U.S. Department of Energy grant number de-sc0010107. FSQ thanks CNPq grants 303817/2018 - 6 and 421952/2018 - 0, and ICTP-SAIFR FAPESP grant 2016/01343 - 7 for the financial support. FQ ans CS are supported by MEC and UFRN, and thank the grant FAPESP 2015/15897-1 for financial support. This work was supported by the Serrapilheira Institute (grant number Serra-191231613).

\section{A Anomaly Freedom}

In this appendix we describe how to find the conditions for anomaly freedom, showed in Eqs.(2.4)-(2.8) when we include a new $U(1)_{X}$ Abelian gauge symmetry:

- $\left[S U(3)_{c}\right]^{2} U(1)_{X}$ :

$$
\mathcal{A}=\operatorname{Tr}\left[\left\{\frac{\lambda^{a}}{2}, \frac{\lambda^{b}}{2}\right\} Y_{R}^{\prime}\right]-\operatorname{Tr}\left[\left\{\frac{\lambda^{a}}{2}, \frac{\lambda^{b}}{2}\right\} Y_{L}^{\prime}\right]
$$

where $\lambda^{a, b}$ are the Gell-Mann matrices related to the symmetry $S U(3)_{c}$ and $Y_{R, L}^{\prime}$ the charges under $U(1)_{X}$. Substituting the Gell-Mann matrices we get,

$$
\mathcal{A} \propto \sum_{\text {quarks }} Y_{R}^{\prime}-\sum_{\text {quarks }} Y_{L}^{\prime}=\left[3 Q_{X}^{u}+3 Q_{X}^{d}\right]-\left[3 \cdot 2 Q_{X}^{q}\right]=0 .
$$

Therefore, the condition for anomaly freedom is:

$$
Q_{X}^{u}+Q_{X}^{d}-2 Q_{X}^{q}=0 .
$$


- $\left[S U(2)_{L}\right]^{2} U(1)_{X}$ :

$$
\mathcal{A}=-\operatorname{Tr}\left[\left\{\frac{\sigma^{a}}{2}, \frac{\sigma^{b}}{2}\right\} Y_{L}^{\prime}\right] \propto-\sum Y_{L}^{\prime}=-\left[2 Q_{X}^{l}+3 \cdot 2 Q_{X}^{q}\right]=0 .
$$

where $\sigma^{a, b}$ are the Pauli matrices related to the $S U(2)_{L}$ symmetry. Therefore, the condition between the $U(1)_{X}$ charges is given by,

$$
Q_{X}^{l}=-3 Q_{X}^{q} .
$$

- $\left[U(1)_{Y}\right]^{2} U(1)_{X}$ :

$$
\mathcal{A}=\operatorname{Tr}\left[\left\{Y_{R}, Y_{R}\right\} Y_{R}^{\prime}\right]-\operatorname{Tr}\left[\left\{Y_{L}, Y_{L}\right\} Y_{L}^{\prime}\right] \propto \sum Y_{R}^{2} Y_{R}^{\prime}-\sum Y_{L}^{2} Y_{L}^{\prime}
$$

where $Y_{R, L}$ are the charges under $U(1)_{Y}$. Substituting the $Y_{R, L}$ charges we found,

$$
\begin{aligned}
\mathcal{A} & \propto\left[(-2)^{2} Q_{X}^{e}+3\left(\frac{4}{3}\right)^{2} Q_{X}^{u}+3\left(-\frac{2}{3}\right)^{2} Q_{X}^{d}\right]+ \\
& -\left[2(-1)^{2} Q_{X}^{l}+3 \cdot 2\left(\frac{1}{3}\right)^{2} Q_{X}^{q}\right]=0 .
\end{aligned}
$$

Therefore, under the anomaly freedom condition, we get the following relation between the $U(1)_{X}$ charges:

$$
6 Q_{X}^{e}+8 Q_{X}^{u}+2 Q_{X}^{d}-3 Q_{X}^{l}-Q_{X}^{q}=0 .
$$

- $U(1)_{Y}\left[U(1)_{X}\right]^{2}$ :

$$
\mathcal{A}=\operatorname{Tr}\left[\left\{Y_{R}^{\prime}, Y_{R}^{\prime}\right\} Y_{R}\right]-\operatorname{Tr}\left[\left\{Y_{L}^{\prime}, Y_{L}^{\prime}\right\} Y_{L}\right] \propto \sum Y_{R} Y_{R}^{\prime 2}-\sum Y_{L} Y_{L}^{\prime 2},
$$

substituting the $U(1)_{Y}$ charges, we get,

$$
\begin{aligned}
\mathcal{A} & \propto\left[(-2)\left(Q_{X}^{e}\right)^{2}+3\left(\frac{4}{3}\right)\left(Q_{X}^{u}\right)^{2}+3\left(-\frac{2}{3}\right)\left(Q_{X}^{d}\right)^{2}\right]+ \\
& -\left[2(-1)\left(Q_{X}^{l}\right)^{2}+3 \cdot 2\left(\frac{1}{3}\right)\left(Q_{X}^{q}\right)^{2}\right]=0 .
\end{aligned}
$$

Therefore, we find the following relations between the $U(1)_{X}$ charges:

$$
-\left(Q_{X}^{e}\right)^{2}+2\left(Q_{X}^{u}\right)^{2}-\left(Q_{X}^{d}\right)^{2}+\left(Q_{X}^{l}\right)^{2}-\left(Q_{X}^{q}\right)^{2}=0 .
$$

- $\left[U(1)_{X}\right]^{3}$ : The last case provides:

$$
\mathcal{A}=\operatorname{Tr}\left[\left\{Y_{R}^{\prime}, Y_{R}^{\prime}\right\} Y_{R}^{\prime}\right]-\operatorname{Tr}\left[\left\{Y_{L}^{\prime}, Y_{L}^{\prime}\right\} Y_{L}^{\prime}\right] \propto \sum Y_{R}^{\prime 3}-\sum Y_{L}^{\prime 3},
$$

where substituting the $U(1)_{X}$ charges, we get

$$
\mathcal{A} \propto\left[\left(Q_{X}^{e}\right)^{3}+3\left(Q_{X}^{u}\right)^{3}+3\left(Q_{X}^{d}\right)^{3}\right]-\left[2\left(Q_{X}^{l}\right)^{3}+3 \cdot 2\left(Q_{X}^{q}\right)^{3}\right]=0,
$$

which gives the following relation:

$$
\left(Q_{X}^{e}\right)^{3}+3\left(Q_{X}^{u}\right)^{3}+3\left(Q_{X}^{d}\right)^{3}-2\left(Q_{X}^{l}\right)^{3}-6\left(Q_{X}^{q}\right)^{3}=0 .
$$

Using the relations described above, it is possible to construct several anomaly free models when we have an extra $U(1)_{X}$ gauge symmetry, as discussed in [49]. 


\section{References}

[1] Planck Collaboration, N. Aghanim et. al., Planck 2018 results. VI. Cosmological parameters, 1807.06209.

[2] G. Arcadi, M. Dutra, P. Ghosh, M. Lindner, Y. Mambrini, M. Pierre, S. Profumo, and F. S. Queiroz, The waning of the WIMP? A review of models, searches, and constraints, Eur. Phys. J. C 78 (2018), no. 3 203, [1703.07364].

[3] R. K. Leane, T. R. Slatyer, J. F. Beacom, and K. C. Ng, GeV-scale thermal WIMPs: Not even slightly ruled out, Phys. Rev. D 98 (2018), no. 2 023016, [1805.10305].

[4] Super-Kamiokande Collaboration, S. Fukuda et. al., Constraints on neutrino oscillations using 1258 days of Super-Kamiokande solar neutrino data, Phys. Rev. Lett. 86 (2001) 5656-5660, [hep-ex/0103033].

[5] SNO Collaboration, Q. Ahmad et. al., Direct evidence for neutrino flavor transformation from neutral current interactions in the Sudbury Neutrino Observatory, Phys. Rev. Lett. 89 (2002) 011301, [nucl-ex/0204008].

[6] KamLAND Collaboration, S. Abe et. al., Precision Measurement of Neutrino

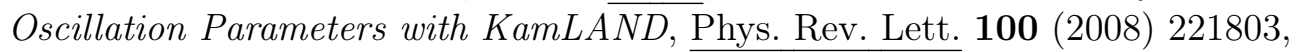
[0801.4589].

[7] T2K Collaboration, K. Abe et. al., Indication of Electron Neutrino Appearance from an Accelerator-produced Off-axis Muon Neutrino Beam, Phys. Rev. Lett. 107 (2011) 041801, [1106.2822].

[8] Double Chooz Collaboration, Y. Abe et. al., Indication of Reactor $\bar{\nu}_{e}$ Disappearance in the Double Chooz Experiment, Phys. Rev. Lett. 108 (2012) 131801, [1112.6353].

[9] Daya Bay Collaboration, F. An et. al., Observation of electron-antineutrino disappearance at Daya Bay, Phys. Rev. Lett. 108 (2012) 171803, [1203.1669].

[10] RENO Collaboration, J. Ahn et. al., Observation of Reactor Electron Antineutrino Disappearance in the RENO Experiment, Phys. Rev. Lett. 108 (2012) 191802, [1204.0626].

[11] MINOS Collaboration, P. Adamson et. al., Electron neutrino and antineutrino appearance in the full MINOS data sample, Phys. Rev. Lett. 110 (2013), no. 17 171801, [1301.4581].

[12] Topical Conveners: K.N. Abazajian, J.E. Carlstrom, A.T. Lee Collaboration, K. Abazajian et. al., Neutrino Physics from the Cosmic Microwave Background and Large Scale Structure, Astropart. Phys. 63 (2015) 66-80, [1309.5383].

[13] M. Aoki, S. Kanemura, and O. Seto, Neutrino mass, Dark Matter and Baryon Asymmetry via TeV-Scale Physics without Fine-Tuning, Phys. Rev. Lett. 102 (2009) 051805, [0807.0361].

[14] T. Nomura and H. Okada, Hidden U(1) gauge symmetry realizing a neutrinophilic two-Higgs-doublet model with dark matter, Phys. Rev. D 97 (2018), no. 7 075038, [1709.06406].

[15] T. Nomura and H. Okada, Neutrinophilic two Higgs doublet model with dark matter 
under an alternative $U(1)_{B-L}$ gauge symmetry, Eur. Phys. J. C 78 (2018), no. 3 189, [1708.08737].

[16] H. Cai, T. Nomura, and H. Okada, A neutrino mass model with hidden U(1) gauge symmetry, Nucl. Phys. B 949 (2019) 114802, [1812.01240].

[17] J. Gehrlein and M. Pierre, A testable hidden-sector model for Dark Matter and neutrino masses, JHEP 02 (2020) 068, [1912.06661].

[18] D. Van Loi, P. Van Dong, and D. Van Soa, Neutrino mass and dark matter from an approximate $B-L$ symmetry, 1911.04902.

[19] S. Singirala, R. Mohanta, S. Patra, and S. Rao, Majorana Dark Matter, Massless Goldstone and Neutrino Mass in a New B - L Model, Springer Proc. Phys. 234 (2019) 315-321.

[20] P. Di Bari, Neutrino masses, leptogenesis and dark matter, in Prospects in Neutrino Physics, 4, 2019. 1904.11971.

[21] S. Mishra, S. Singirala, and S. Sahoo, Scalar dark matter, Neutrino mass, Leptogenesis and rare $B$ decays in a $U(1)_{B-L}$ model, 1908.09187.

[22] W. Chao, M. Gonderinger, and M. J. Ramsey-Musolf, Higgs Vacuum Stability, Neutrino Mass, and Dark Matter, Phys. Rev. D 86 (2012) 113017, [1210.0491].

[23] D. Restrepo, A. Rivera, and W. Tangarife, Singlet-Doublet Dirac Dark Matter and Neutrino Masses, Phys. Rev. D 100 (2019), no. 3 035029, [1906.09685].

[24] P. Fileviez Pérez, C. Murgui, and A. D. Plascencia, Neutrino-Dark Matter Connections in Gauge Theories, Phys. Rev. D 100 (2019), no. 3 035041, [1905.06344].

[25] A. Das, S. Goswami, K. Vishnudath, and T. Nomura, Constraining a general U(1)' inverse seesaw model from vacuum stability, dark matter and collider, $\underline{\text { Phys. Rev. D }}$ 101 (2020), no. 5 055026, [1905.00201].

[26] C. Jaramillo, M. Lindner, and W. Rodejohann, Seesaw neutrino dark matter by freeze-out, 2004.12904.

[27] J. A. Dror, D. Dunsky, L. J. Hall, and K. Harigaya, Sterile Neutrino Dark Matter in Left-Right Theories, 2004.09511.

[28] P. Van Dong, Flipping principle for neutrino mass and dark matter, 2003.13276.

[29] B. Shakya, Sterile Neutrino Dark Matter from Freeze-In, Mod. Phys. Lett. A 31 (2016), no. 06 1630005, [1512.02751].

[30] T. Asaka, S. Blanchet, and M. Shaposhnikov, The nuMSM, dark matter and neutrino masses, Phys. Lett. B 631 (2005) 151-156, [hep-ph/0503065].

[31] S. Dodelson and L. M. Widrow, Sterile-neutrinos as dark matter, Phys. Rev. Lett. 72 (1994) 17-20, [hep-ph/9303287].

[32] A. Dolgov and S. Hansen, Massive sterile neutrinos as warm dark matter, Astropart. Phys. 16 (2002) 339-344, [hep-ph/0009083].

[33] K. Abazajian, G. M. Fuller, and M. Patel, Sterile neutrino hot, warm, and cold dark matter, Phys. Rev. D 64 (2001) 023501, [astro-ph/0101524]. 
[34] K. Abazajian, Production and evolution of perturbations of sterile neutrino dark matter, Phys. Rev. D 73 (2006) 063506, [astro-ph/0511630].

[35] T. Asaka, M. Laine, and M. Shaposhnikov, Lightest sterile neutrino abundance within the nuMSM, JHEP 01 (2007) 091, [hep-ph/0612182]. [Erratum: JHEP 02, 028 (2015)].

[36] A. Kusenko, Sterile neutrinos: The Dark side of the light fermions, Phys. Rept. 481 (2009) 1-28, [0906. 2968].

[37] K. N. Abazajian, Sterile neutrinos in cosmology, Phys. Rept. 711-712 (2017) 1-28, [1705.01837].

[38] A. Boyarsky, M. Drewes, T. Lasserre, S. Mertens, and O. Ruchayskiy, Sterile Neutrino Dark Matter, Prog. Part. Nucl. Phys. 104 (2019) 1-45, [1807.07938].

[39] X.-D. Shi and G. M. Fuller, A New dark matter candidate: Nonthermal sterile neutrinos, Phys. Rev. Lett. 82 (1999) 2832-2835, [astro-ph/9810076].

[40] A. Schneider, Astrophysical constraints on resonantly produced sterile neutrino dark matter, JCAP 04 (2016) 059, [1601.07553].

[41] M. Laine and M. Shaposhnikov, Sterile neutrino dark matter as a consequence of nuMSM-induced lepton asymmetry, JCAP 06 (2008) 031, [0804.4543].

[42] K. Petraki and A. Kusenko, Dark-matter sterile neutrinos in models with a gauge singlet in the Higgs sector, Phys. Rev. D 77 (2008) 065014, [0711.4646].

[43] J. König, A. Merle, and M. Totzauer, keV Sterile Neutrino Dark Matter from Singlet Scalar Decays: The Most General Case, JCAP 11 (2016) 038, [1609.01289].

[44] M. Dutra, M. Lindner, S. Profumo, F. S. Queiroz, W. Rodejohann, and C. Siqueira, MeV Dark Matter Complementarity and the Dark Photon Portal, JCAP 03 (2018) 037, [1801.05447].

[45] N. Okada and O. Seto, Higgs portal dark matter in the minimal gauged $U(1)_{B-L}$ model, Phys. Rev. D 82 (2010) 023507, [1002.2525].

[46] N. Okada and S. Okada, $Z^{\prime}$-portal right-handed neutrino dark matter in the minimal $U(1)_{X}$ extended Standard Model, Phys. Rev. D 95 (2017), no. 3 035025, [1611.02672].

[47] N. Okada and S. Okada, $Z_{B L}^{\prime}$ portal dark matter and LHC Run-2 results, Phys. Rev. D 93 (2016), no. 7 075003, [1601.07526].

[48] S. Okada, $Z^{\prime}$ Portal Dark Matter in the Minimal B - L Model, Adv. High Energy Phys. 2018 (2018) 5340935, [1803.06793].

[49] M. D. Campos, D. Cogollo, M. Lindner, T. Melo, F. S. Queiroz, and W. Rodejohann, Neutrino Masses and Absence of Flavor Changing Interactions in the 2HDM from Gauge Principles, JHEP 08 (2017) 092, [1705.05388].

[50] D. A. Camargo, L. Delle Rose, S. Moretti, and F. S. Queiroz, Collider bounds on 2-Higgs doublet models with U (1) X gauge symmetries, Phys. Lett. B 793 (2019) 150-160, [1805.08231].

[51] S. Blasi, V. Brdar, and K. Schmitz, Fingerprint of Low-Scale Leptogenesis in the Primordial Gravitational-Wave Spectrum, 2004.02889. 
[52] T. Lee, A Theory of Spontaneous T Violation, Phys. Rev. D 8 (1973) 1226-1239.

[53] H. E. Haber and G. L. Kane, The Search for Supersymmetry: Probing Physics Beyond the Standard Model, Phys. Rept. 117 (1985) 75-263.

[54] G. Branco, P. Ferreira, L. Lavoura, M. Rebelo, M. Sher, and J. P. Silva, Theory and phenomenology of two-Higgs-doublet models, Phys. Rept. 516 (2012) 1-102, [1106.0034].

[55] S. Bertolini, Quantum Effects in a Two Higgs Doublet Model of the Electroweak Interactions, Nucl. Phys. B 272 (1986) 77-98.

[56] K. Babu and E. Ma, Bounds on Higgs Boson Masses in a Two Doublet Extension of the Standard Model, Phys. Rev. D 31 (1985) 2861. [Erratum: Phys.Rev.D 33, 3471 (1986)].

[57] M. Sher, Electroweak Higgs Potentials and Vacuum Stability, Phys. Rept. 179 (1989) 273-418.

[58] V. D. Barger, J. Hewett, and R. Phillips, New Constraints on the Charged Higgs Sector in Two Higgs Doublet Models, Phys. Rev. D 41 (1990) 3421-3441.

[59] P. H. Chankowski, M. Krawczyk, and J. Zochowski, Implications of the precision data for very light Higgs boson scenario in 2HDM(II), Eur. Phys. J. C 11 (1999) 661-672, [hep-ph/9905436].

[60] J. F. Gunion and H. E. Haber, The CP conserving two Higgs doublet model: The Approach to the decoupling limit, Phys. Rev. D 67 (2003) 075019, [hep-ph/0207010].

[61] A. Barroso, P. Ferreira, R. Santos, M. Sher, and J. P. Silva, 2HDM at the LHC - the story so far, in 1st Toyama International Workshop on Higgs as a Probe of New Physics 2013, 4, 2013. 1304.5225.

[62] T. G. Rizzo, $b \rightarrow s \gamma$ in the Two Higgs Doublet Model, Phys. Rev. D 38 (1988) 820.

[63] M. Ciuchini, G. Degrassi, P. Gambino, and G. Giudice, Next-to-leading QCD corrections to $B \rightarrow X_{s} \gamma$ : Standard model and two Higgs doublet model, Nucl. Phys. B $\mathbf{5 2 7}$ (1998) 21-43, [hep-ph/9710335].

[64] M. Lindner, M. Platscher, and F. S. Queiroz, A Call for New Physics : The Muon Anomalous Magnetic Moment and Lepton Flavor Violation, Phys. Rept. 731 (2018) $1-82,[1610.06587]$.

[65] A. Davidson, M. Koca, and K. C. Wali, Minimal anomaly-free electroweak model for several generations, Phys. Rev. D 20 (Sep, 1979) 1195-1206.

[66] P. Ferreira and J. P. Silva, Abelian symmetries in the two-Higgs-doublet model with fermions, Phys. Rev. D 83 (2011) 065026, [1012.2874].

[67] I. Ivanov and C. Nishi, Abelian symmetries of the $\mathrm{N}$-Higgs-doublet model with Yukawa interactions, JHEP 11 (2013) 069, [1309.3682].

[68] H. Serôdio, Yukawa sector of Multi Higgs Doublet Models in the presence of Abelian symmetries, Phys. Rev. D 88 (2013), no. 5 056015, [1307.4773].

[69] W.-C. Huang, Y.-L. S. Tsai, and T.-C. Yuan, G2HDM : Gauged Two Higgs Doublet Model, JHEP 04 (2016) 019, [1512.00229]. 
[70] A. Crivellin, G. D'Ambrosio, and J. Heeck, Explaining $h \rightarrow \mu^{ \pm} \tau^{\mp}, B \rightarrow K^{*} \mu^{+} \mu^{-}$and $B \rightarrow K \mu^{+} \mu^{-} / B \rightarrow K e^{+} e^{-}$in a two-Higgs-doublet model with gauged $L_{\mu}-L_{\tau}$, Phys. Rev. Lett. 114 (2015) 151801, [1501.00993].

[71] W. Wang and Z.-L. Han, Global U(1) ${ }_{L}$ Breaking in Neutrinophilic 2HDM: From LHC Signatures to X-Ray Line, Phys. Rev. D 94 (2016), no. 5 053015, [1605.00239].

[72] L. Delle Rose, S. Khalil, and S. Moretti, Explanation of the $17 \mathrm{MeV}$ Atomki anomaly in a U(1) -extended two Higgs doublet model, Phys. Rev. D 96 (2017), no. 11 115024, [1704.03436].

[73] S.-P. Li, X.-Q. Li, Y.-D. Yang, and X. Zhang, $R_{D^{(*)}}, R_{K^{(*)}}$ and neutrino mass in the 2HDM-III with right-handed neutrinos, JHEP 09 (2018) 149, [1807.08530].

[74] S.-P. Li, X.-Q. Li, and Y.-D. Yang, Muon g- 2 in a U(1)-symmetric Two-Higgs-Doublet Model, Phys. Rev. D 99 (2019), no. 3 035010, [1808.02424].

[75] S. Iguro and Y. Omura, Status of the semileptonic $B$ decays and muon g-2 in general 2HDMs with right-handed neutrinos, JHEP 05 (2018) 173, [1802.01732].

[76] G. Arcadi, M. Lindner, J. Martins, and F. S. Queiroz, New Physics Probes: Atomic Parity Violation, Polarized Electron Scattering and Neutrino-Nucleus Coherent Scattering, 1906.04755.

[77] C.-T. Huang, R. Ramos, V. Q. Tran, Y.-L. S. Tsai, and T.-C. Yuan, Consistency of Gauged Two Higgs Doublet Model: Gauge Sector, JHEP 09 (2019) 048, [1905. 02396].

[78] D. A. Camargo, M. Klasen, and S. Zeinstra, Discovering heavy U(1)-gauged Higgs bosons at the HL-LHC, 1903.02572.

[79] A. Ordell, R. Pasechnik, H. Serôdio, and F. Nottensteiner, Classification of anomaly-free 2HDMs with a gauged U(1) symmetry, Phys. Rev. D 100 (2019), no. 11 115038, [1909.05548].

[80] P. Ko, Y. Omura, and C. Yu, Higgs phenomenology in Type-I 2HDM with $U(1)_{H}$ Higgs gauge symmetry, JHEP 01 (2014) 016, [1309.7156].

[81] D. A. Camargo, A. G. Dias, T. B. de Melo, and F. S. Queiroz, Neutrino Masses in a Two Higgs Doublet Model with a U(1) Gauge Symmetry, JHEP 04 (2019) 129, [1811.05488].

[82] D. Cogollo, R. D. Matheus, T. B. de Melo, and F. S. Queiroz, Type I + II Seesaw in a Two Higgs Doublet Model, Phys. Lett. B 797 (2019) 134813, [1904.07883].

[83] S. Baek, A. Das, and T. Nomura, Scalar dark matter search from the extended

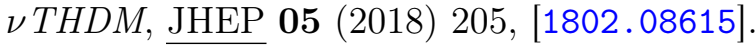

[84] C.-R. Chen, Y.-X. Lin, V. Q. Tran, and T.-C. Yuan, Pair production of Higgs bosons at the LHC in gauged 2HDM, Phys. Rev. D 99 (2019), no. 7 075027, [1810.04837].

[85] D. A. Camargo, M. D. Campos, T. B. de Melo, and F. S. Queiroz, A Two Higgs Doublet Model for Dark Matter and Neutrino Masses, Phys. Lett. B 795 (2019) 319-326, [1901.05476].

[86] C.-R. Chen, Y.-X. Lin, C. S. Nugroho, R. Ramos, Y.-L. S. Tsai, and T.-C. Yuan, Complex scalar dark matter in the gauged two-Higgs-doublet model, Phys. Rev. D 101 (2020), no. 3 035037, [1910.13138]. 
[87] C. H. Nam, Collider phenomenology in emergent $U(1)_{X}$ extension of the Standard Model, 2001.02421.

[88] D. Borah, D. Nanda, N. Narendra, and N. Sahu, Right-handed neutrino dark matter with radiative neutrino mass in gauged B - L model, Nucl. Phys. B 950 (2020) 114841, [1810.12920].

[89] J. Casas and A. Ibarra, Oscillating neutrinos and $\mu \rightarrow e, \gamma$, Nucl. Phys. B 618 (2001) 171-204, [hep-ph/0103065].

[90] Particle Data Group Collaboration, M. Tanabashi et. al., Review of Particle Physics, Phys. Rev. D 98 (2018), no. 3030001.

[91] B. Holdom, Two U(1)'s and Epsilon Charge Shifts, Phys. Lett. B 166 (1986) 196-198.

[92] C. Cheung, J. T. Ruderman, L.-T. Wang, and I. Yavin, Kinetic Mixing as the Origin of Light Dark Scales, Phys. Rev. D 80 (2009) 035008, [0902.3246].

[93] M. Lindner, Y. Mambrini, T. B. de Melo, and F. S. Queiroz, XENON1T Anomaly: A Light $Z^{\prime}, 2006.14590$.

[94] G. Arcadi, M. D. Campos, M. Lindner, A. Masiero, and F. S. Queiroz, Dark sequential Z' portal: Collider and direct detection experiments, Phys. Rev. D 97 (2018), no. 4 043009, [1708.00890].

[95] D. Mahanta and D. Borah, Fermion dark matter with $N_{2}$ leptogenesis in minimal scotogenic model, JCAP 11 (2019) 021, [1906.03577].

[96] G. Belanger, F. Boudjema, A. Pukhov, and A. Semenov, MicrOMEGAs 2.0: A Program to calculate the relic density of dark matter in a generic model, Comput. Phys. Commun. 176 (2007) 367-382, [hep-ph/0607059].

[97] G. Belanger, F. Boudjema, A. Pukhov, and A. Semenov, micrOMEGAs 2.0.7: A program to calculate the relic density of dark matter in a generic model, Comput. Phys. Commun. 177 (2007) 894-895.

[98] G. F. Giudice, E. W. Kolb, and A. Riotto, Largest temperature of the radiation era and its cosmological implications, Phys. Rev. D 64 (2001) 023508, [hep-ph/0005123].

[99] G. Arcadi and P. Ullio, Accurate estimate of the relic density and the kinetic decoupling in non-thermal dark matter models, Phys. Rev. D 84 (2011) 043520, [1104.3591].

[100] M. Drees and F. Hajkarim, Dark Matter Production in an Early Matter Dominated Era, JCAP 02 (2018) 057, [1711.05007].

[101] F. D'Eramo, N. Fernandez, and S. Profumo, When the Universe Expands Too Fast: Relentless Dark Matter, JCAP 05 (2017) 012, [1703.04793].

[102] P. Chanda, S. Hamdan, and J. Unwin, Reviving $Z$ and Higgs Mediated Dark Matter Models in Matter Dominated Freeze-out, JCAP 01 (2020) 034, [1911.02616].

[103] P. Arias, N. Bernal, A. Herrera, and C. Maldonado, Reconstructing Non-standard Cosmologies with Dark Matter, JCAP 10 (2019) 047, [1906.04183].

[104] D. Mahanta and D. Borah, TeV Scale Leptogenesis with Dark Matter in Non-standard Cosmology, JCAP 04 (2020), no. 04 032, [1912.09726]. 
[105] M. Kawasaki, K. Kohri, and N. Sugiyama, MeV scale reheating temperature and thermalization of neutrino background, Phys. Rev. D 62 (2000) 023506, [astro-ph/0002127].

[106] S. Hannestad, What is the lowest possible reheating temperature?, Phys. Rev. D 70 (2004) 043506, [astro-ph/0403291].

[107] F. De Bernardis, L. Pagano, and A. Melchiorri, New constraints on the reheating temperature of the universe after WMAP-5, Astropart. Phys. 30 (2008) 192-195.

[108] P. de Salas, M. Lattanzi, G. Mangano, G. Miele, S. Pastor, and O. Pisanti, Bounds on very low reheating scenarios after Planck, Phys. Rev. D 92 (2015), no. 12 123534, [1511.00672].

[109] A. L. Fitzpatrick, W. Haxton, E. Katz, N. Lubbers, and Y. Xu, The Effective Field Theory of Dark Matter Direct Detection, JCAP 1302 (2013) 004, [1203.3542].

[110] A. L. Fitzpatrick, W. Haxton, E. Katz, N. Lubbers, and Y. Xu, Model Independent Direct Detection Analyses, 1211.2818.

[111] N. Anand, A. L. Fitzpatrick, and W. C. Haxton, Weakly interacting massive particle-nucleus elastic scattering response, Phys. Rev. C89 (2014), no. 6 065501, [1308.6288].

[112] M. Cirelli, E. Del Nobile, and P. Panci, Tools for model-independent bounds in direct dark matter searches, JCAP 1310 (2013) 019, [1307.5955].

[113] M. Duerr, F. Kahlhoefer, K. Schmidt-Hoberg, T. Schwetz, and S. Vogl, How to save the WIMP: global analysis of a dark matter model with two s-channel mediators, JHEP 09 (2016) 042, [1606.07609].

[114] F. D'Eramo, B. J. Kavanagh, and P. Panci, You can hide but you have to run: direct detection with vector mediators, JHEP 08 (2016) 111, [1605.04917].

[115] A. Berlin, D. Hooper, and S. D. McDermott, Simplified Dark Matter Models for the Galactic Center Gamma-Ray Excess, Phys. Rev. D 89 (2014), no. 11 115022, [1404.0022].

[116] Fermi-LAT Collaboration, M. Ackermann et. al., Searching for Dark Matter Annihilation from Milky Way Dwarf Spheroidal Galaxies with Six Years of Fermi Large Area Telescope Data, Phys. Rev. Lett. 115 (2015), no. 23 231301, [1503.02641].

[117] H. Liu, G. W. Ridgway, and T. R. Slatyer, Code package for calculating modified cosmic ionization and thermal histories with dark matter and other exotic energy injections, Phys. Rev. D 101 (2020), no. 2 023530, [1904.09296].

[118] F. Kahlhoefer, K. Schmidt-Hoberg, T. Schwetz, and S. Vogl, Implications of unitarity and gauge invariance for simplified dark matter models, JHEP 02 (2016) 016, [1510.02110].

[119] G. Arcadi, M. Lindner, Y. Mambrini, M. Pierre, and F. S. Queiroz, GUT Models at Current and Future Hadron Colliders and Implications to Dark Matter Searches, Phys. Lett. B 771 (2017) 508-514, [1704.02328].

[120] ATLAS Collaboration, G. Aad et. al., Search for new resonances in mass distributions of jet pairs using $1 \overline{39 \mathrm{fb}^{-1}}$ of pp collisions at $\sqrt{\mathrm{s}}=13 \mathrm{TeV}$ with the ATLAS detector, 1910.08447. 
[121] ATLAS Collaboration, M. Aaboud et. al., Search for low-mass resonances decaying into two jets and produced in association with a photon using pp collisions at $\sqrt{s}=13$ TeV with the ATLAS detector, Phys. Lett. B 795 (2019) 56-75, [1901.10917].

[122] CMS Collaboration, A. M. Sirunyan et. al., Search for low mass vector resonances decaying into quark-antiquark pairs in proton-proton collisions at $\sqrt{s}=13 \mathrm{TeV}$, Phys. Rev. D 100 (2019), no. 11 112007, [1909.04114].

[123] ATLAS Collaboration, G. Aad et. al., Search for high-mass dilepton resonances using $139 \mathrm{fb}^{-1}$ of pp collision data collected at $\sqrt{\mathrm{s}}=13 \mathrm{TeV}$ with the ATLAS detector, Phys. Lett. B 796 (2019) 68-87, [1903.06248].

[124] ATLAS Collaboration, M. Aaboud et. al., Search for heavy particles decaying into a top-quark pair in the fully hadronic final state in pp collisions at $\sqrt{s}=13$ TeV with the ATLAS detector, Phys. Rev. D 99 (2019), no. 9 092004, [1902.10077].

[125] ATLAS Collaboration, M. Aaboud et. al., Search for dark matter and other new phenomena in events with an energetic jet and large missing transverse momentum using the ATLAS detector, JHEP 01 (2018) 126, [1711.03301].

[126] S. Porsev, K. Beloy, and A. Derevianko, Precision determination of weak charge of ${ }^{133}$ Cs from atomic parity violation, Phys. Rev. D 82 (2010) 036008, [1006.4193].

[127] G. Arcadi, A. Djouadi, and M. Raidal, Dark Matter through the Higgs portal, Phys. Rept. 842 (2020) 1-180, [1903.03616].

[128] BaBar Collaboration, B. Aubert et. al., Direct CP, Lepton Flavor and Isospin Asymmetries in the Decays $B \rightarrow \overline{K^{(*)} \ell^{+}} \ell^{-}$, Phys. Rev. Lett. 102 (2009) 091803, [0807.4119].

[129] BaBar Collaboration, G. Eigen, Branching Fraction and CP Asymmetry Measurements in Inclusive $B \rightarrow X_{s} \ell^{+} \ell^{-}$and $B \rightarrow X_{s} \gamma$ Decays from BaBar, Nucl. Part. Phys. Proc. 273-275 (2016) 1459-1464, [1503.02294].

[130] TEXONO Collaboration, H. Li et. al., Limit on the electron neutrino magnetic moment from the Kuo-Sheng reactor neutrino experiment, Phys. Rev. Lett. 90 (2003) 131802, [hep-ex/0212003].

[131] TEXONO Collaboration, H. Wong et. al., A Search of Neutrino Magnetic Moments with a High-Purity Germanium Detector at the Kuo-Sheng Nuclear Power Station, Phys. Rev. D 75 (2007) 012001, [hep-ex/0605006].

[132] LSND Collaboration, L. Auerbach et. al., Measurement of electron - neutrino electron elastic scattering, Phys. Rev. D 63 (2001) 112001, [hep-ex/0101039].

[133] TEXONO Collaboration, M. Deniz et. al., Measurement of Nu(e)-bar -Electron Scattering Cross-Section with a CsI (Tl) Scintillating Crystal Array at the Kuo-Sheng Nuclear Power Reactor, Phys. Rev. D 81 (2010) 072001, [0911.1597].

[134] G. Bellini et. al., Precision measurement of the 7Be solar neutrino interaction rate in Borexino, Phys. Rev. Lett. 107 (2011) 141302, [1104.1816].

[135] A. Beda, E. Demidova, A. Starostin, V. Brudanin, V. Egorov, D. Medvedev, M. Shirchenko, and T. Vylov, GEMMA experiment: Three years of the search for the neutrino magnetic moment, Phys. Part. Nucl. Lett. 7 (2010) 406-409, [0906.1926]. 